\title{
Adverse Event Profile of Pyrimethamine-Based Therapy in Toxoplasmosis: A Systematic Review
}

\author{
Ruben R. Ben-Harari ${ }^{1}$ (D) Elizabeth Goodwin ${ }^{1} \cdot$ Julio Casoy $^{1}$
}

Published online: 6 September 2017

(C) The Author(s) 2017. This article is an open access publication

\begin{abstract}
Introduction Approximately a third of the population worldwide is chronically infected with Toxoplasma gondii. Pyrimethamine-based regimens are recommended for the treatment of toxoplasmosis.

Objective The aim was to evaluate the safety profile of pyrimethamine-based treatment for the three main Toxoplasma manifestations: toxoplasmic encephalitis (TE), ocular toxoplasmosis, and congenital toxoplasmosis.

Methods PubMed, Cochrane Library, and Google Scholar databases were searched through August 1, 2016. Randomized, observational, prospective/retrospective, and cohort studies were eligible. Thirty-one studies were included with a total of 2975 patients. Of these, 13 were in congenital toxoplasmosis $(n=929), 11$ in ocular toxoplasmosis $(n=1284)$, and seven in TE $(n=687)$. Across manifestations, adverse event (AE)-related treatment discontinuation and/or change in therapy involved $\leq 37 \%$ of patients and occurred in $>55 \%$ of studies: $100 \%$ for ocular toxoplasmosis, $57.1 \%$ for $\mathrm{TE}$, and $61.5 \%$ for congenital toxoplasmosis. The most commonly observed AEs were bone marrow suppression, dermatologic, and gastrointestinal (GI). The prevalence of bone marrow suppressionrelated AEs was $\leq 50 \%$ in congenital toxoplasmosis, $\leq 42.7 \%$ in TE, and $\leq 9.0 \%$ in ocular toxoplasmosis. The frequency of GI and dermatologic AEs were $\leq 100$ and $\leq 11.1 \%$, respectively, for ocular toxoplasmosis, $\leq 10.7$ and $\leq 17.9 \%$ for $\mathrm{TE}$, and $\leq 10.8$ and $\leq 2.1 \%$ for congenital
\end{abstract}

Ruben R. Ben-Harari

ruben@turingpharma.com

1 Turing Pharmaceuticals, 600 Third Avenue, 10th Floor, New York, NY 10016, USA toxoplasmosis. Steven-Johnson syndrome was reported in two patients with ocular toxoplasmosis and one with TE. Conclusion The AE profile associated with pyrimethamine-based treatments differed by each manifestation of toxoplasmosis and within a given manifestation. Hematologic AEs occurred across all manifestations indicating the importance of monitoring the blood of patients administered pyrimethamine-based regimens.

\section{Key Points}

The types of adverse events associated with pyrimethamine-based treatment of toxoplasmosis differed across the three clinical manifestations of the disease: ocular toxoplasmosis, toxoplasmic encephalitis, and congenital toxoplasmosis.

The differences in adverse event profiles among the three manifestations and between studies within each manifestation are likely due to differences in patient populations, dosing regimens, study protocols, and pharmacokinetics of pyrimethamine.

Hematologic adverse events were observed across all manifestations, highlighting the importance of monitoring blood in patients receiving pyrimethamine plus other drugs to treat toxoplasmosis. 


\section{Introduction}

Toxoplasmosis is caused by the protozoan parasite Toxoplasma gondii (T. gondii), an obligate intracellular parasite capable of infecting a wide range of hosts and many different types of host cells [1]. Approximately a third of the population worldwide is chronically infected with $T$. gondii [2]. In the USA, it is estimated that about $22 \%$ of the population $\leq 12$ years are infected with the parasite [3]. The seroprevalence of $T$. gondii varies across the world. For example, T. gondii seroprevalence is $>60 \%$ in Brazil, the Philippines, and Madagascar; 40-60\% in Egypt, Argentina, and several European countries, including France, Germany, and Poland; and $20-40 \%$ in Australia, Chile, Saudi Arabia, Iran, and the USA [4]. Infections occur through consuming food (including meat products) or water contaminated with cat feces, motherto-child (congenital/vertical) and iatrogenic (transplanted organs and blood transfusion) transmission [3]. The three most important manifestations of $T$. gondii infection are toxoplasmic encephalitis (TE), ocular toxoplasmosis, and congenital toxoplasmosis $[3,5,6]$. Benign lymphadenopathy is also observed in immunocompetent individuals and often resolves without therapy [3]. Each year in the USA an estimated 4839 persons develop symptomatic ocular toxoplasmosis [7], an average of 20,258 hospitalizations are associated with TE [8], and an estimated one in 10,000 live births has congenital toxoplasmosis [9].

The recommended first-line treatment for toxoplasmosis is a combination therapy based on pyrimethamine, sulfadiazine, and leucovorin or folinic acid [3, 10]. Pyrimethamine is an anti-parasitic medication that has been available for over 60 years and is indicated for the treatment of toxoplasmosis. Pyrimethamine and sulfadiazine are thought to act synergistically to treat toxoplasmosis by inhibiting $T$. gondii proliferation and survival through inhibiting the folate metabolic pathway [3, 10-12]. The drugs inhibit dihydrofolate reductase (DHFR) and dihydropteroate synthase (DHPS), respectively, and consequently block the synthesis of tetrahydrofolate, which is required by the parasite for DNA synthesis [12, 13]. Pyrimethamine-associated adverse events (AEs) are mainly related to its inhibition of folic acid metabolism in tissues with high metabolic activity (e.g., epithelium and bone marrow) [14]. Pyrimethamine-related bone marrow suppression is reversible and usually recovers following cessation or reduction of therapy and/or addition of folinic acid or leucovorin $[15,16]$. Leucovorin and folinic acid are reduced forms of folic acid and are readily converted to tetrahydrofolate [17], hence, bypassing the inhibition of DHFR by pyrimethamine.
AEs associated with pyrimethamine often result in change or discontinuation of therapy, and consequently impact treatment outcomes. To date, several systematic reviews have focused on the efficacy and safety of different treatment regimens in treating TE in adults with HIV or AIDS [18-21], congenital toxoplasmosis [18], and ocular toxoplasmosis $[18,22]$. However, none have assessed the overall safety of pyrimethamine-based treatment regimens across all three major manifestations. The purpose of this systematic review is to provide a comprehensive summary of the safety of pyrimethamine-based therapy in treating TE, ocular toxoplasmosis, and congenital toxoplasmosis. Due to the small number of randomized trials in toxoplasmosis, relative to the number of studies with other designs, our analysis allowed for inclusion of studies of varying design, such as single-arm, observational cohorts, randomized, and retrospective studies.

\section{Methods}

\subsection{Search Strategy}

The review was performed in accordance with PRISMA guidelines. PubMed, Google Scholar, and Cochrane databases were searched through August 1, 2017 using the following search terms: pyrimethamine, Daraprim, Fansidar, Metakelfin, Fansimef, 5-(4-chlorophenyl)-6-ethyl-2,4pyrimidinediamine, encephalitis, cerebral, toxoplasmosis, toxoplasmic, congenital, and gondii. A manual search of references from recent reviews and relevant published original studies was also performed to identify additional studies. Studies eligible for inclusion were randomized, observational prospective and retrospective, and cohort studies. Included studies reported AEs for pyrimethaminebased treatment regimens, had patient populations of ten or more, and were published in English in peer-reviewed journals. For TE, only studies performed from 1996 to August 1, 2017 (independent of publication date) were included. The decision to review only TE papers performed during this time period was based on the introduction of highly active antiretroviral therapy (HAART), which in 1996 became widely available, resulting in a dramatic decrease in mortality among patients with AIDS; mortality declined from 29.4 per 100 person-years in 1995 to 8.8 per 100 person-years in the second quarter of 1997 [23]. Several papers have indicated that toxoplasmosis treatment response in patients with HIV/AIDS and TE, such as relapse rate, differed between the pre-HAART and post-HAART periods [24-26]. In addition, HAART is currently the standard of care for the treatment of HIV/AIDS and, hence, the AE profiles reported following 1996 for this patient population 
are clinically most relevant. Letters, comments, editorials, proceedings, case reports, correspondence, personal communications, in vitro studies, and preclinical studies were excluded. Study eligibility and data extraction were performed by two independent reviewers, and a third reviewer was consulted to adjudicate disagreements.

\subsection{Data Extraction and Quality Assessment}

The following information was extracted: first author, age, gender, treatment regimens, duration of treatment, duration of follow-up, and AEs. AEs are those that were observed following treatment and, depending upon the study, were reported as AEs, safety, side effects, and/or toxicities.

The quality of the included studies was evaluated using the National Heart, Lung, and Blood Institute of the National Institutes of Health Study Quality Assessment Tools for observational cohort and cross-sectional studies, before-after (pre-post) studies with no control group, and controlled intervention studies [27]. Each report was rated as poor, fair, or high quality.

\section{Results}

\subsection{Search Results}

The search identified 2504 records, and an additional 174 records were identified through other sources (Fig. 1). Of the 2678 records initially identified, 2385 were excluded after removal of duplicates and initial title and abstract screening. Following a full-text review, 262 additional studies were excluded for being case reports, evaluating only drug stability, not reporting safety, describing only a study protocol, not presenting efficacy of a pyrimethaminebased regimen, duplication of another publication, being a TE study performed prior to 1996 (see Sect. 2.1, for explanation for including only post-1996 studies), or being a survey. Thirty-one studies were included in the systematic review. The number of studies for each major manifestation were 13 for congenital toxoplasmosis, 11 for ocular toxoplasmosis, and seven for TE. The number of patients totaled 2975.

\subsection{Quality Assessment}

The majority of included studies obtained quality scores of medium to low due to high risk of bias with no blinding of participants, personnel, and outcome assessment being the main issues.

\subsection{Summary of Adverse Events in Ocular Toxoplasmosis}

The search identified 11 studies that reported AEs associated with pyrimethamine-based treatment in ocular toxoplasmosis (Table 1). The 11 studies included a total of 1284 patients (range 19-303), of which 870 received a
Fig. 1 Search flow diagram

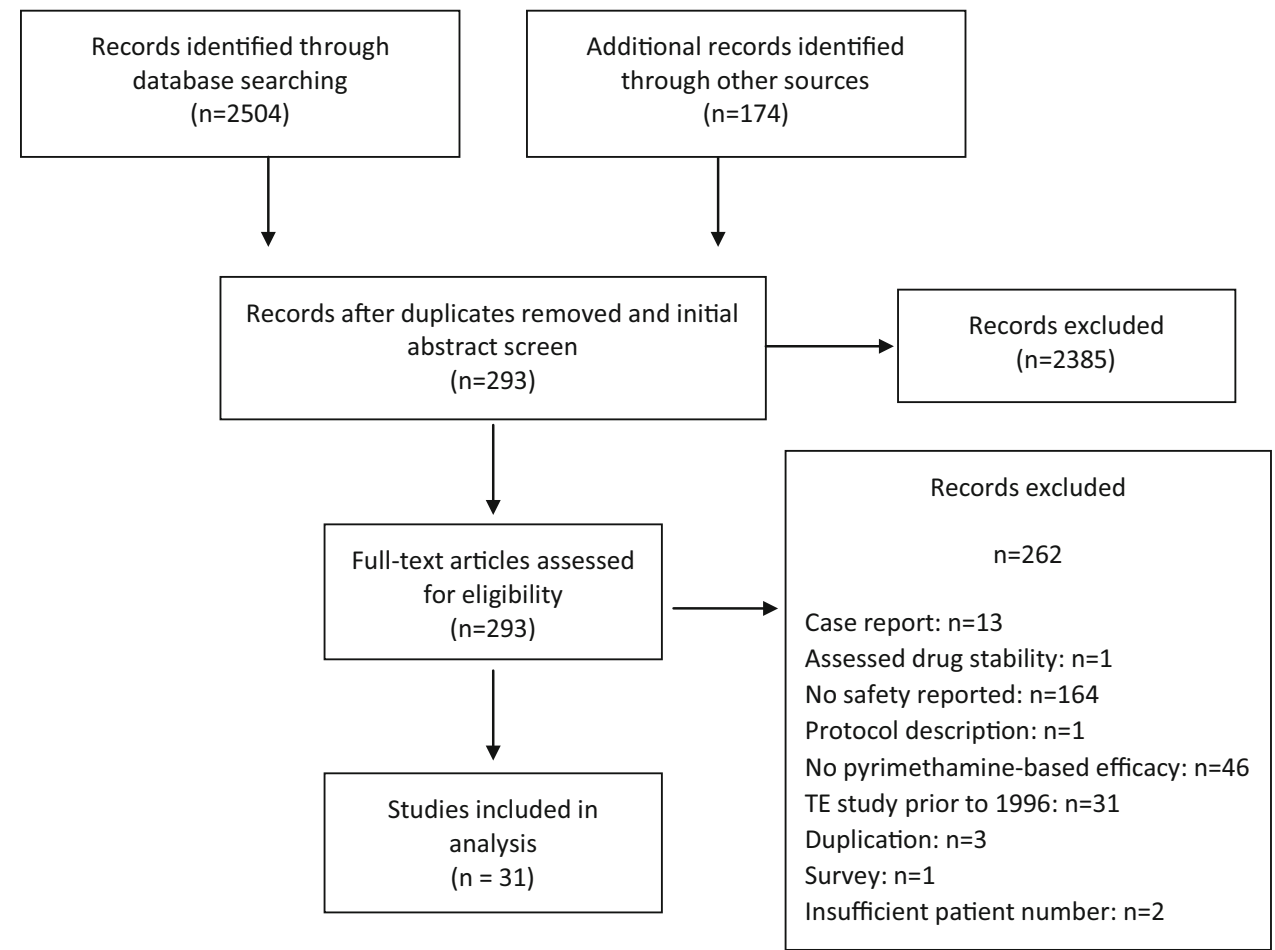














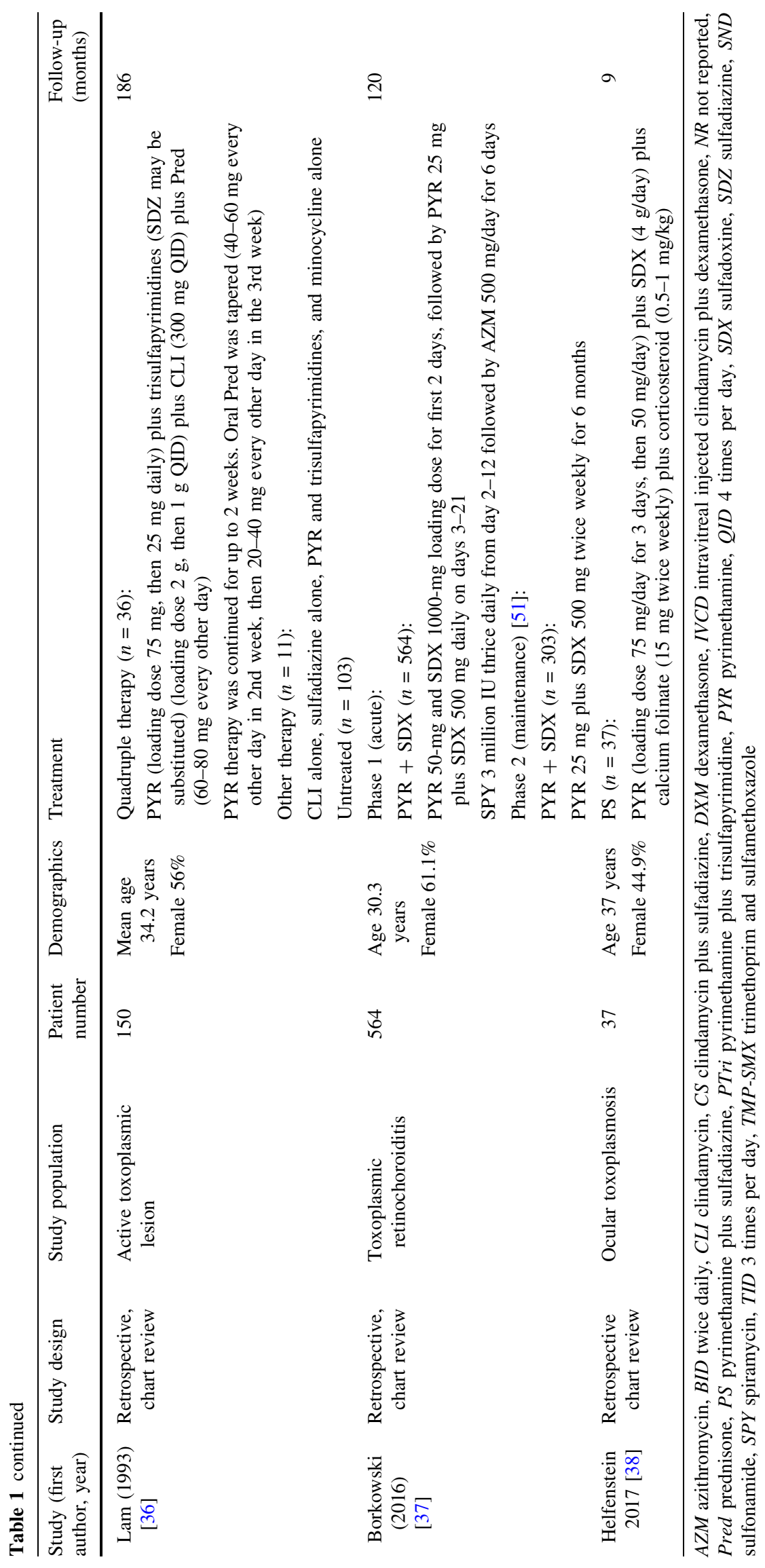




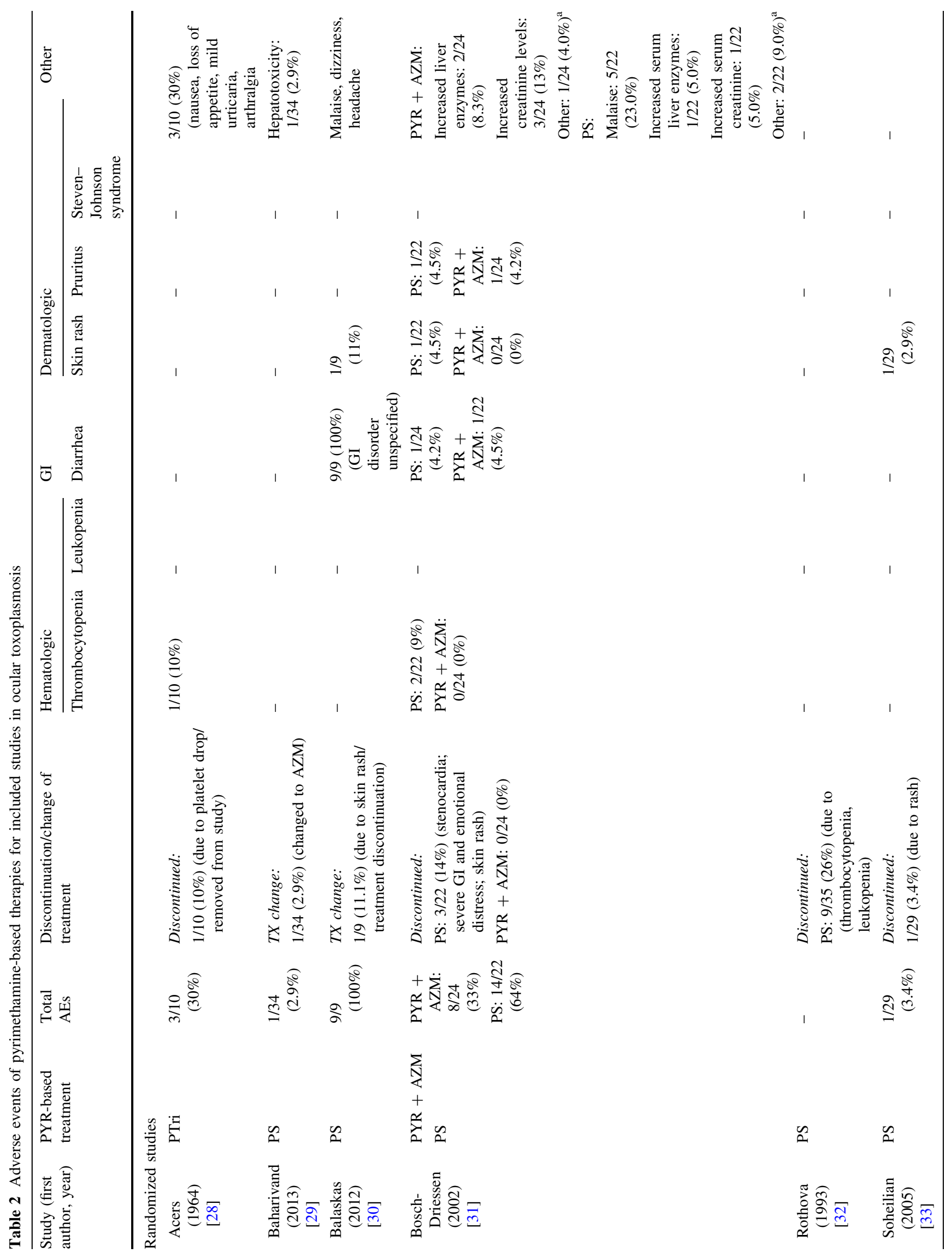




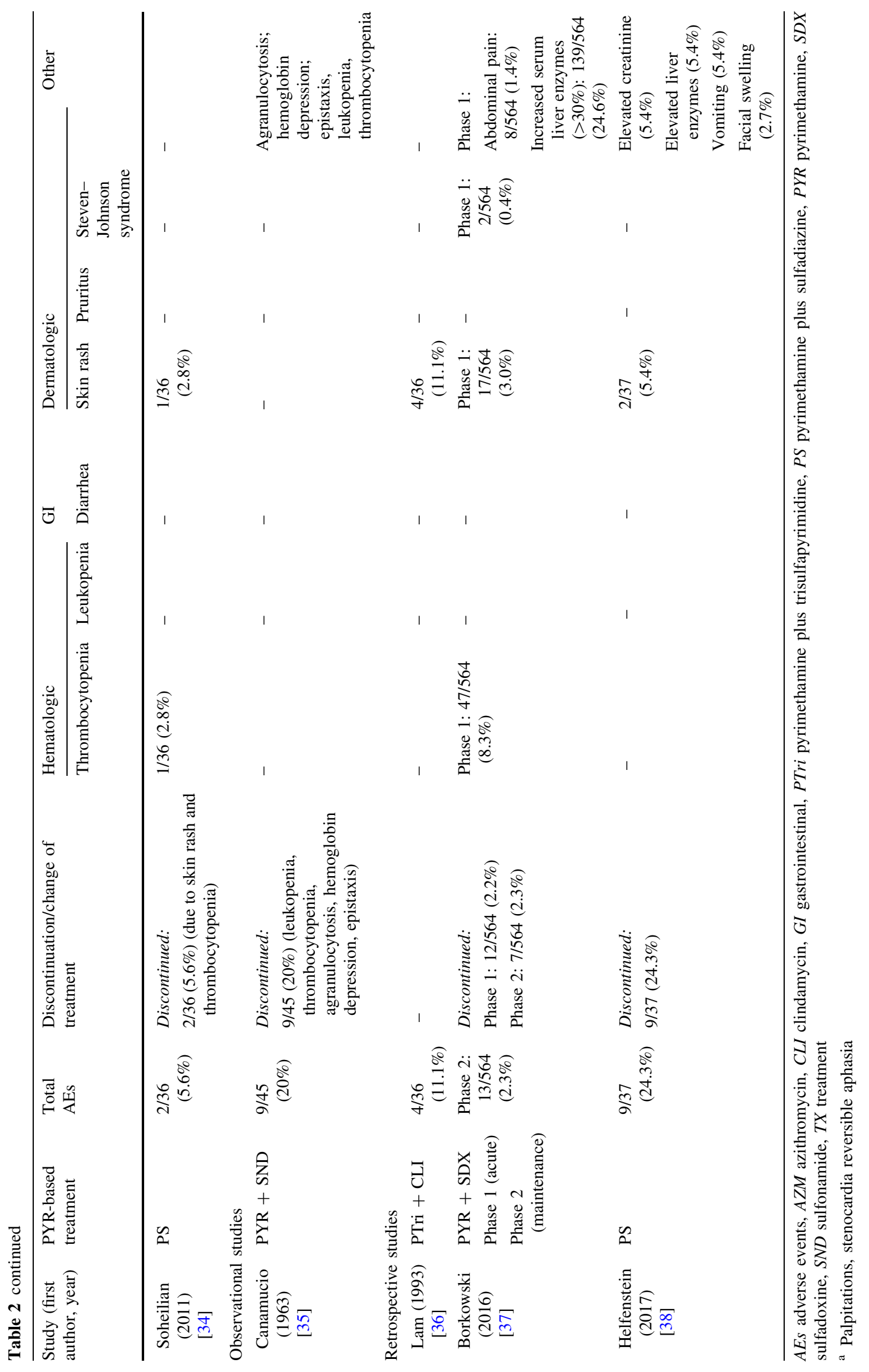


treatment regimen containing pyrimethamine. None of the patients were immunocompromised. Seven studies were randomized prospective studies, which compared at least two different treatment regimens [28-34], one study was a prospective cohort study [35], and three were retrospective chart reviews [36-38] in design. All studies included pyrimethamine-based regimens that contained a sulfonamide, except for the study of Bosch-Driessen et al. [31], which contained one treatment arm with pyrimethamine plus azithromycin. Ocular toxoplasmosis was diagnosed both by clinical (e.g., presence of vitreous inflammation, retinochoroiditis, retinal scars) and serologic analyses of the presence of anti-Toxoplasma antibodies ( $\operatorname{IgG}$ and $\operatorname{IgM}$ ).

The dose of pyrimethamine varied across studies. Four studies did not report coadministering folinic acid or leucovorin with pyrimethamine [28, 35-37]. Ten of the studies first administered a loading dose of pyrimethamine ranging from 50 to $200 \mathrm{mg} /$ day [29, 31-38]. Other treatment regimens evaluated were spiramycin, clindamycin, azithromycin, and trimethoprim-sulfamethoxazole (TMPSMX). Duration of acute treatment varied among studies, ranging from 2 to about 10 weeks. The range of follow-up among the studies was from $<3$ months to 16 years.

The total number of patients reporting AEs varied across studies, ranging from 2.3 to $100 \%$ (Table 2). Each of the 11 studies reported discontinuation or change in treatment due to AEs (range 0-26\%).

Hematologic AEs indicative of bone marrow suppression were reported in five $(45.5 \%)$ of the 11 studies [28, 34, 35, 37, 39], and included patients with thrombocytopenia $(n=52)$. Leukopenia and agranulocytosis were listed as toxic reactions in one study [35]. No neutropenia was reported. Prevalence of these AEs reported among the studies ranged from 2.8 to $10.0 \%$.

A number of non-hematologic AEs were reported across studies. Gastrointestinal (GI)-related AEs were reported in four studies [28, 31, 37, 38], and included diarrhea, GI distress, nausea, vomiting, and loss of appetite. Eight studies $(72.7 \%)$ reported dermatologic AEs [28, 30, 31, 33, 34, 36-38], including skin rash, pruritus, and Steven-Johnson syndrome. The most common dermatologic AE was skin rash (range 2.8-11\%), the presence of which was only associated with pyrimethamine plus sulfadiazine treatment. Two patients $(2 / 564 ; 0.4 \%)$ in one study developed Steven-Johnson syndrome and discontinued therapy [37]. The outcomes of these patients are unclear, as the information is not described in the paper.

Altered liver function, including increased liver enzymes, was reported in four studies (36.4\%) $[29,31,37,38]$, with a frequency that ranged from 2.9 to $24.6 \%$, and studies reported increased serum creatinine levels (range 45-13\%) [31, 38]. Other AEs observed in single studies included fever, epistaxis, dizziness, headache, reversible aphasia, palpitations, stenocardia, emotional distress, arthralgia, facial swelling, and malaise. The prevalence of these AEs, where reported, was $\leq 10 \%$ except for malaise, which was reported in one study to have a prevalence of $23 \%$ [31].

\subsection{Summary of Adverse Events in Toxoplasmic Encephalitis}

The search identified seven studies that were performed from 1996 to August 1, 2017 (post-HAART) that reported AEs associated with pyrimethamine-based treatment of TE (Table 3). The seven studies included a total of 687 patients (range 11-323), of which 626 received a treatment regimen containing pyrimethamine for acute and/or maintenance therapy for TE. Two of the studies were randomized [40, 41], one was prospective [42], and four were retrospective [43-46] in design. The patient population in all studies consisted of patients with TE and with HIV infection or AIDS. Acute therapy was evaluated in six studies [40, 42-46] and maintenance treatment in four [40, 41, 45, 46]. The pyrimethamine-based treatment regimens among the studies were heterogeneous. The most common regimen evaluated was pyrimethamine plus sulfadiazine $(n=3)$. Other pyrimethamine-based regimens included atovaquone, TMP-SMX, or sulfadoxine or another sulfonamide. Only two studies did not report coadministering folinic acid or leucovorin [42, 46]. Acute treatment ranged from 2 to 9 weeks. The duration of follow-up, when reported, varied and ranged from 4 to 48 weeks.

Across the studies, the range of total AEs was from 0 to $62.5 \%$ (Table 4). Treatment discontinuation due to AEs ranged from 10.6 to $18.9 \%$. AEs observed in two or more studies included those associated with bone marrow suppression, GI disturbance, rash, and fever (Table 4). Three studies reported rash (range 6.3-10.8\%) [41, 43, 45], two reported nausea and/or vomiting (range 3.4-10.7\%) [40, 41], and two reported fever (range 7.6-10.7\%) [40, 41]. Hematologic AEs that occurred in two or more of the seven studies included neutropenia $(n=2)[40,45]$, thrombocytopenia $(n=2)$ [40, 43], and pancytopenia $(n=2)$ [43, 46]. Among these studies, the frequency of neutropenia ranged from 7.0 to $25 \%$, thrombocytopenia from 7.1 to $32.5 \%$, and pancytopenia from 6.6 to $21.7 \%$. Other AEs associated with bone marrow suppression reported in a single study were leukopenia $(75 / 323 ; 23.2 \%)$ [43], anemia $(138 / 323 ; 42.7 \%)$ [43], hematologic toxicity $(2 / 66 ; 3.0 \%)$ [41], bleeding $(57 / 323 ; 17.6 \%)$ [43], and thrombocytopenia with major bleeding $(4 / 16 ; 25 \%)$ [45].

Non-hematologic AEs reported in single studies included eczema $(19 / 106 ; 17.9 \%)$ [46], Steven-Johnson syndrome $(1 / 16 ; 6.3 \%)$ [45], pain or discomfort $(2 / 28 ; 7.1 \%)$ 







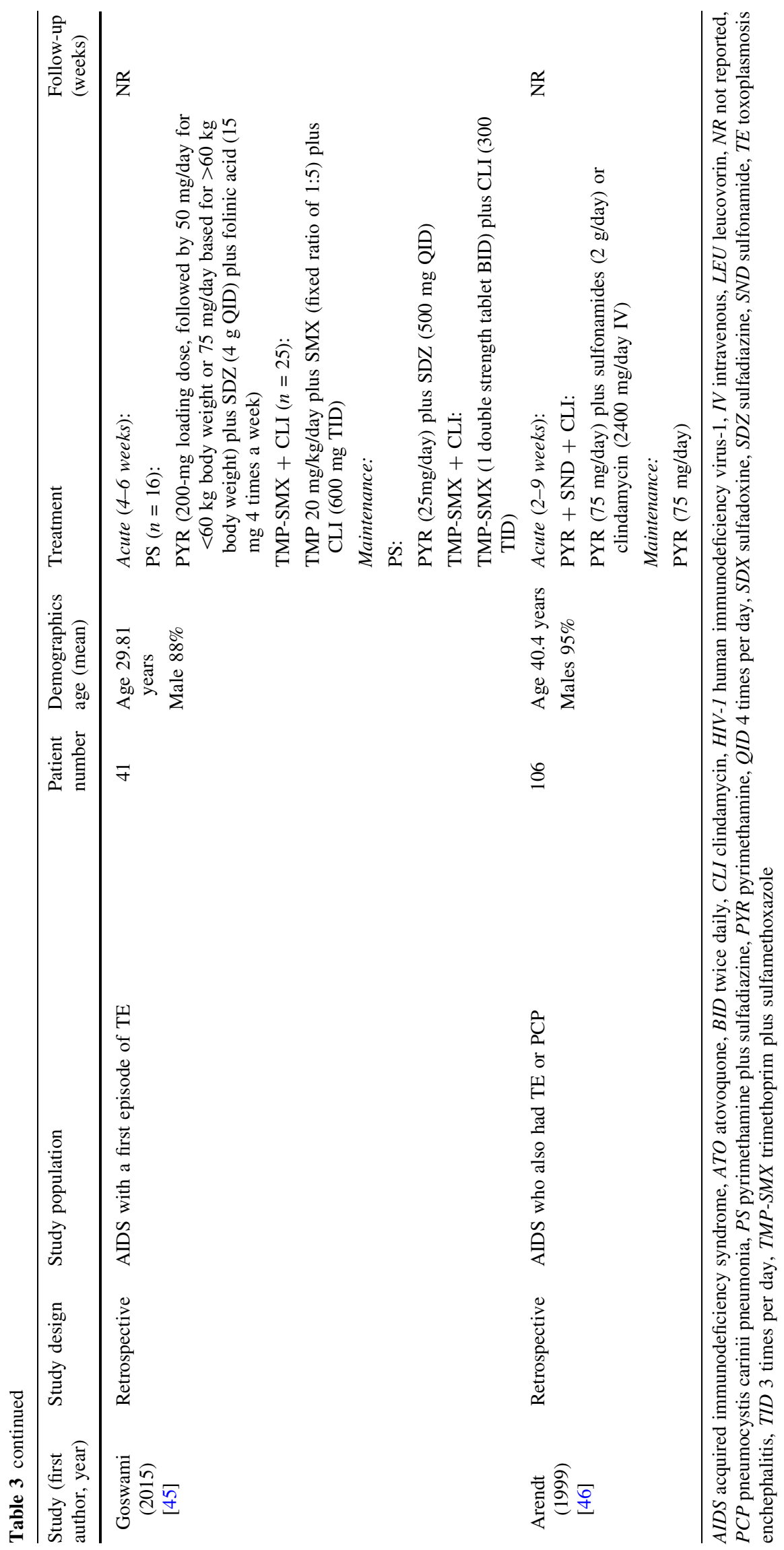




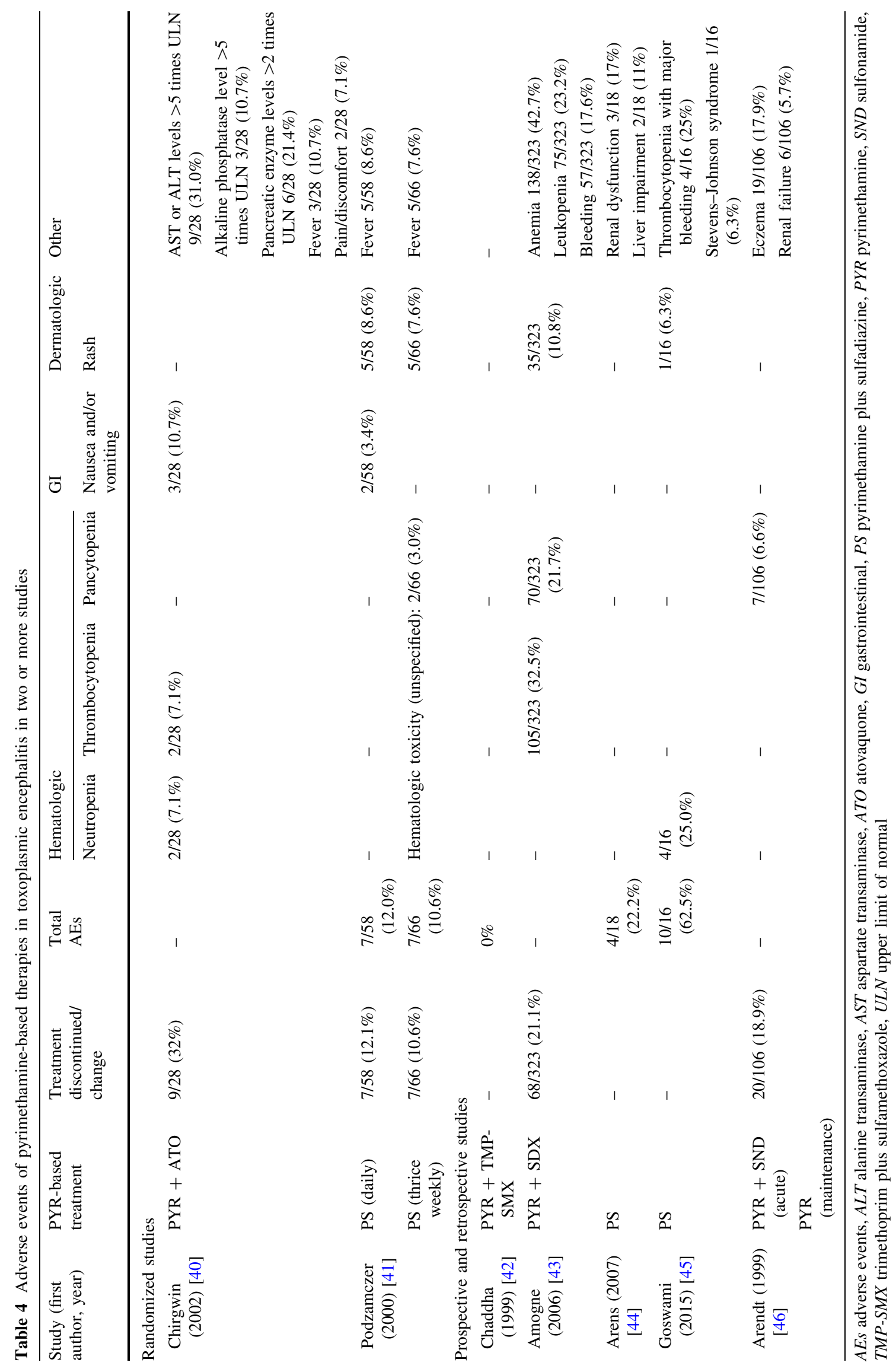









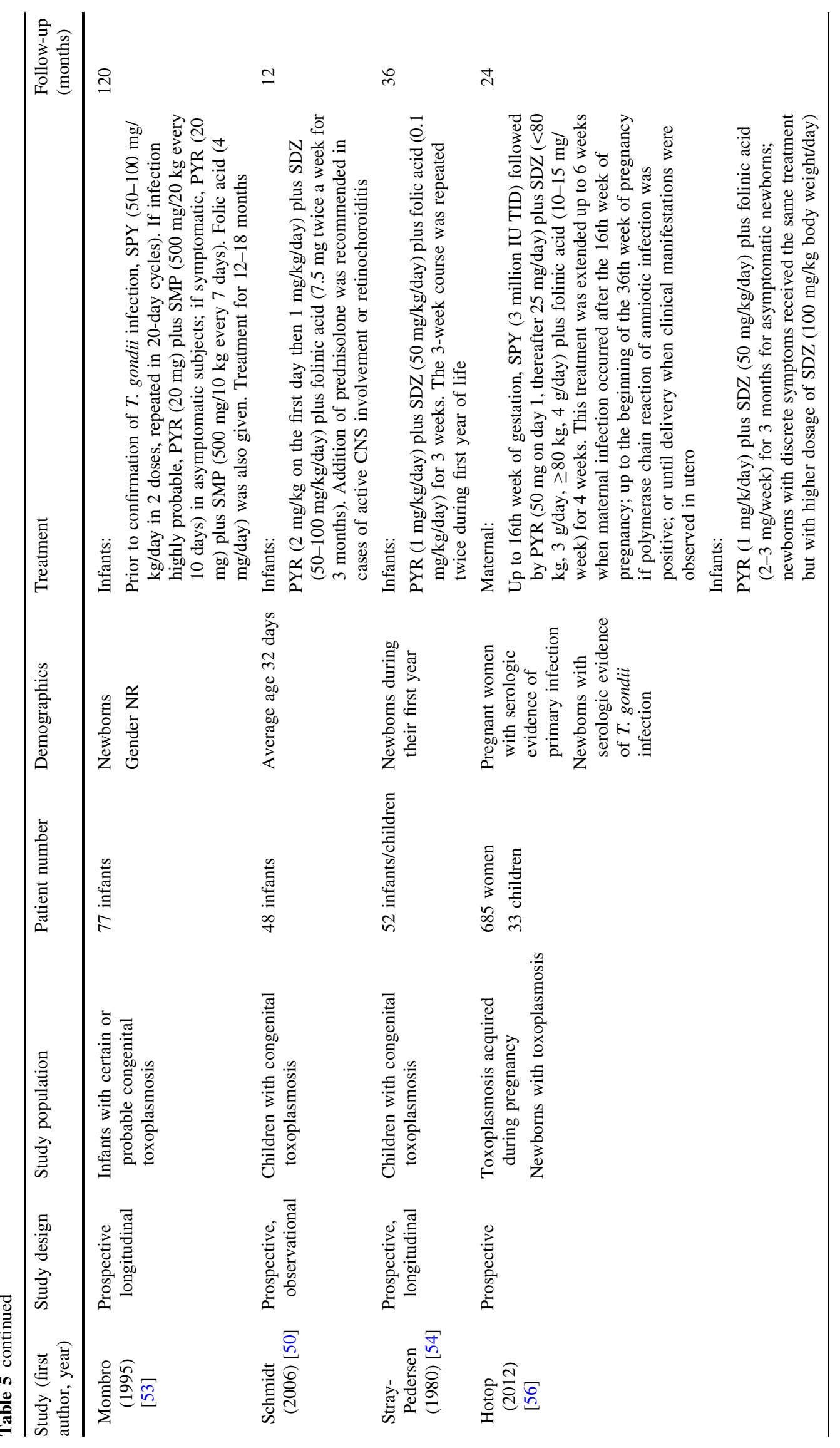




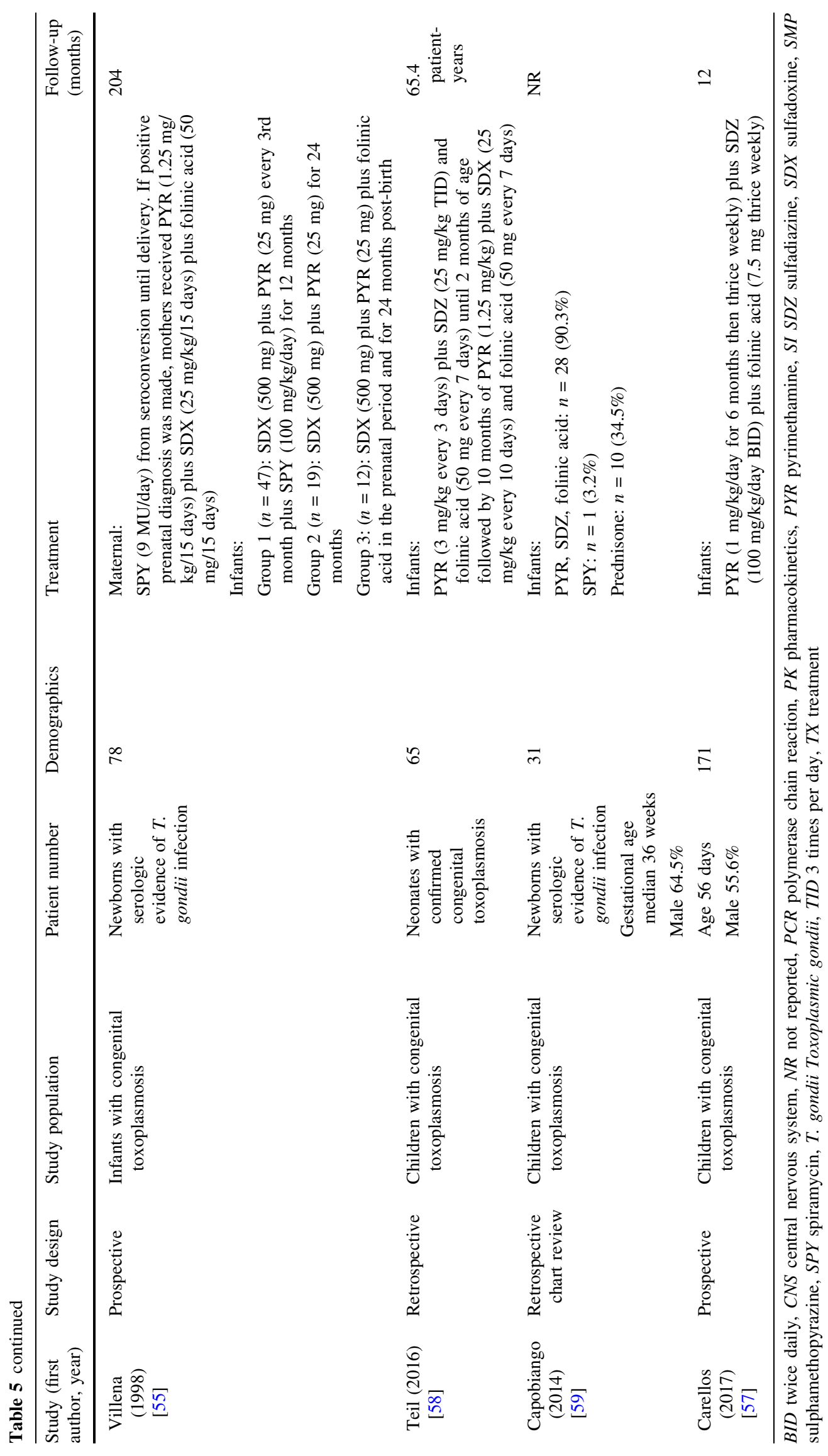




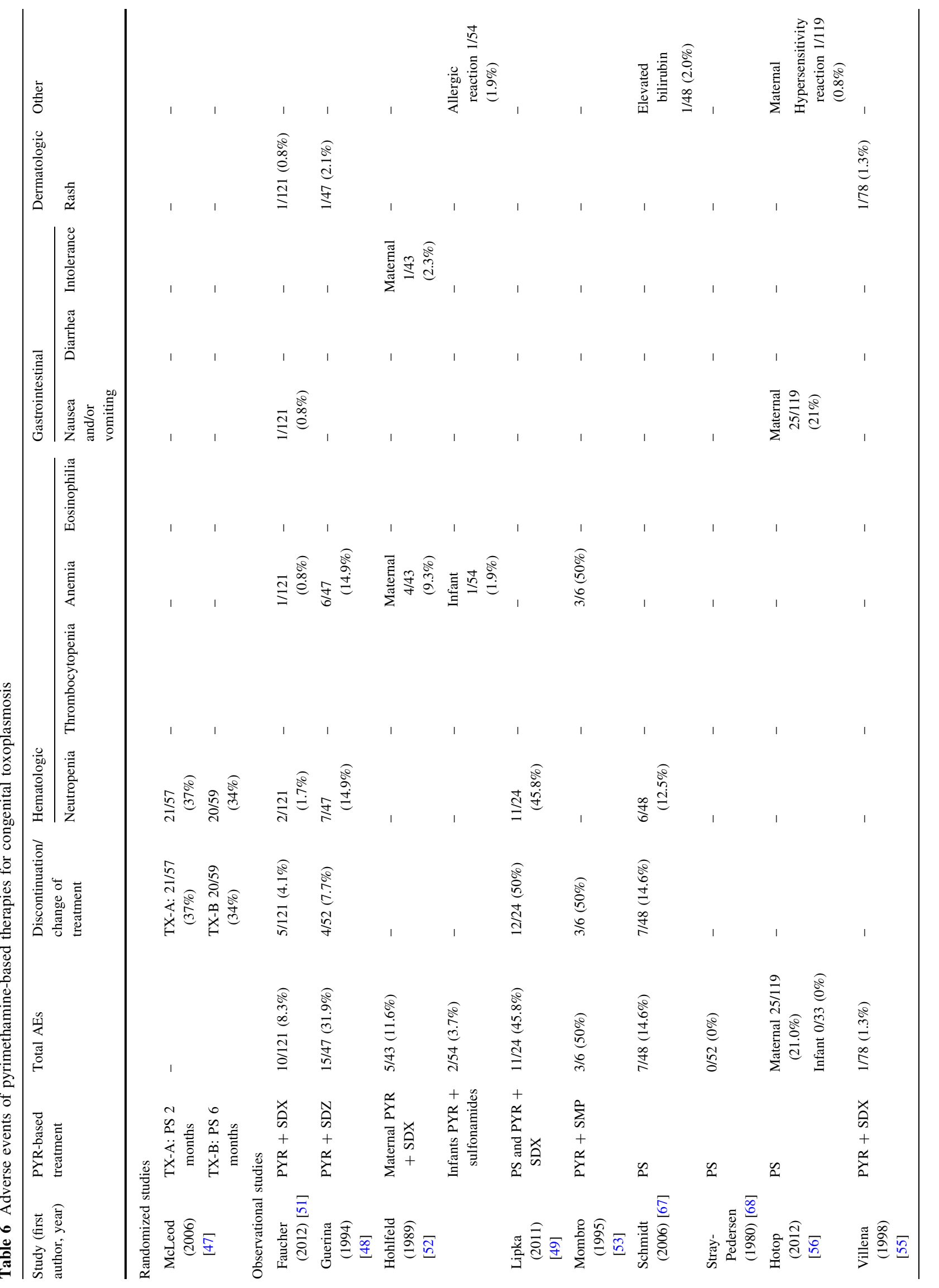




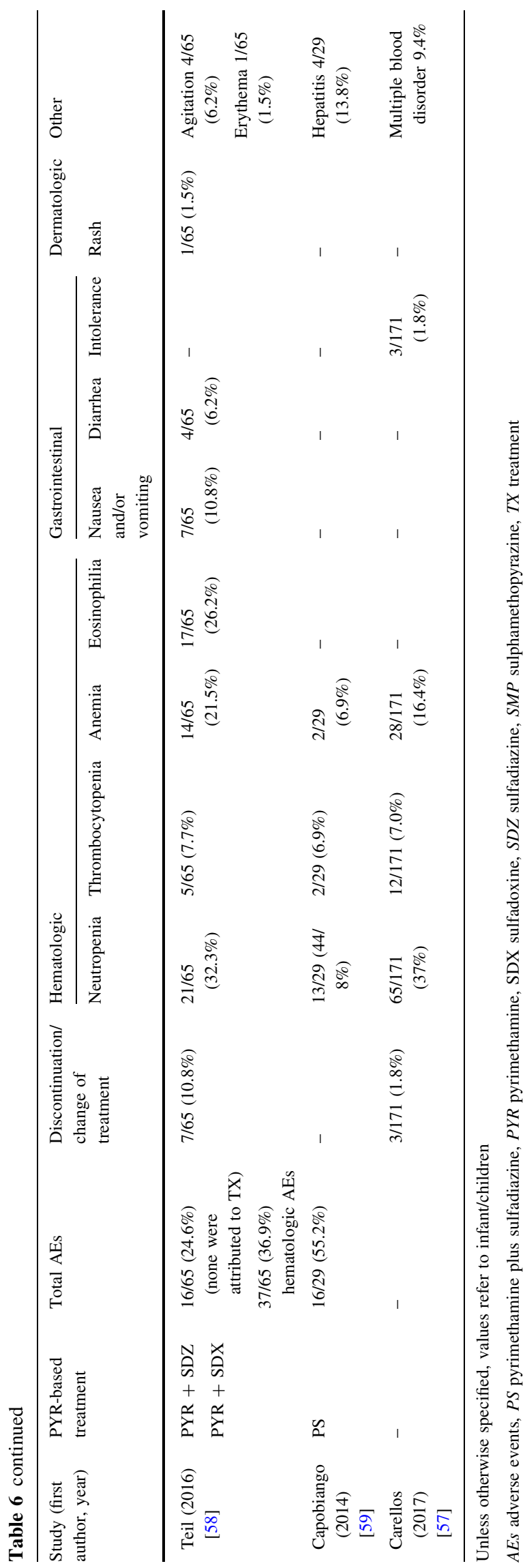

[40], alanine transaminase or aspartate transaminase levels $>5$ times upper limit of normal (ULN) $(9 / 28 ; 32.1 \%)$ [40], alkaline phosphatase levels $>5$ times ULN $(3 / 28 ; 10.7 \%)$ [40], hepatotoxicity $(2 / 18 ; 11.1 \%)$ [44], and pancreatic enzyme levels $>2$ times ULN $(6 / 28 ; 21.4 \%)$ [40]. The outcome of the patient with Steven-Johnson syndrome is not clear as the person was lost to follow-up [45].

\subsection{Summary of Adverse Events in Congenital Toxoplasmosis}

The search identified 13 studies that reported AEs associated with pyrimethamine-based treatment for congenital toxoplasmosis (Table 5). The studies that reported AEs for pregnant mothers and/or infants/children included a total of 929 infants/children with congenital toxoplasmosis (range 24-171) and a total of 737 pregnant mothers (range 52-685). In the studies that described treatment for infants/ children, one was a randomized study [47], ten were prospective [48-57], and two were retrospective [58, 59] in design. The treatment regimens for these studies varied, but all contained pyrimethamine, a sulfonamide, and folic acid, folinic acid, or leucovorin. In the four studies that described maternal treatment $[51,52,55,56]$, mothers were administered spiramycin if infection occurred $<16$ weeks [56] or $<30$ weeks [51], or throughout pregnancy if maternal infection was proven or strongly suspected $[52,55]$. If seroconversion occurred, indicating maternal infection with $T$. gondii, mothers were given a dosing regimen that contained pyrimethamine plus a sulfonamide and folinic acid until delivery $[51,52,55,56]$. In general, the length of treatment for infants was 1 year, with the exception of the study of Schmidt et al. [50], which had a duration of treatment of 3 months. Follow-up among all the studies ranged from 6 months to 20 years.

Eight of the 13 studies (61.5\%) reported discontinuation or change in treatment due to AEs in infants with congenital toxoplasmosis [47, 49-51, 53, 57, 60, 61] (Table 6). AEs associated with bone marrow suppression in infants/ children occurred in ten of the 13 studies (76.9\%) $[47,49-53,57,59-61]$ and in mothers occurred in one of two studies [52]. In infant/children, neutropenia was reported in seven studies $(53.8 \%)$ [47-51, 58, 59], anemia in seven $(53.8 \%)$ [ $48,51-53,57-59]$, thrombocytopenia in three (23.1\%) [57-59], and eosinophilia in one (7.7\%) [58]. Among these studies, the prevalence ranged from 1.7 to $45.8 \%$ for neutropenia, from 0.8 to $50.0 \%$ for anemia, from 6.9 to $7.7 \%$ for thrombocytopenia, and was $26.2 \%$ for eosinophilia. One study reported a frequency of anemia of $9.3 \%$ in mothers treated with pyrimethamine-based therapy [52].

In infants, GI tract-related AEs were reported in three $(23.1 \%)$ of the studies, including nausea and/or vomiting 
Table 7 Summary of treatment discontinuation/change and adverse events by most common type across manifestations

\begin{tabular}{|c|c|c|c|c|}
\hline Manifestation & Discontinued/change in treatment & Bone marrow suppression & Gastrointestinal & Dermatologic \\
\hline \multicolumn{5}{|l|}{ Ocular toxoplasmosis } \\
\hline Frequency by study & $11 / 11(100 \%)$ & $4 / 11(36.4 \%)$ & $4 / 11(36.4 \%)$ & $7 / 11(63.6 \%)$ \\
\hline Prevalence, range & $2.2-26 \%$ & $2.8-9.0 \%$ & $4.2-100 \%$ & $0.3-11.1 \%$ \\
\hline \multicolumn{5}{|c|}{ Toxoplasmic encephalitis } \\
\hline Frequency by study & 4/7 (57.1\%) & $5 / 7(71.4 \%)$ & $2 / 7(28.6 \%)$ & 4/7 (57.1\%) \\
\hline Prevalence, range & $12.1-32.1 \%$ & $6.6-42.7 \%$ & $3.4-10.7 \%$ & $6.3-17.9 \%$ \\
\hline \multicolumn{5}{|c|}{ Congenital toxoplasmosis ${ }^{\mathrm{a}}$} \\
\hline Frequency by study & $8 / 13(61.5 \%)$ & $10 / 13(76.9 \%)$ & $3 / 13(23.1 \%)$ & $4 / 13(30.8 \%)$ \\
\hline Prevalence, range & $1.8-50 \%$ & $0.8-50 \%$ & $0.8-10.8 \%$ & $0.8-2.1 \%$ \\
\hline
\end{tabular}

a Values represent only data from infants

Table 8 Summary of treatment discontinuation/change and adverse events for pyrimethamine plus sulfadiazine and pyrimethamine plus sulfadoxine

\begin{tabular}{|c|c|c|c|c|}
\hline Manifestation & Discontinued/change in treatment & Bone marrow suppression & Gastrointestinal & Dermatologic \\
\hline \multicolumn{5}{|c|}{ Pyrimethamine plus sulfadiazine } \\
\hline Frequency by study & $14 / 19(73.7 \%)$ & $11 / 19(57.9 \%)$ & $6 / 19(31.6 \%)$ & $8 / 19(42.1 \%)$ \\
\hline Prevalence, range & $1.8-50.0 \%$ & $2.8-45.8 \%$ & $1.8-100 \%$ & $1.5-11.0 \%$ \\
\hline \multicolumn{5}{|c|}{ Pyrimethamine plus sulfadoxine } \\
\hline Frequency by study & $5 / 6(83.3 \%)$ & $5 / 6(83.3 \%)$ & $3 / 6(50.0 \%)$ & $5 / 6(83.3 \%)$ \\
\hline Prevalence, range & $2.2-50.0 \%$ & $0.8-45.8 \%$ & $0.8-10.8 \%$ & $0.8-10.8 \%$ \\
\hline
\end{tabular}

Values included for congenital toxoplasmosis were those for infants

$(n=2)$, diarrhea $(n=1)$, and GI intolerance $(n=1)$ $[51,57,58]$. The range in the prevalence of nausea and/or vomiting was $0.8-10.8 \%$, and the incidence of diarrhea was $6.2 \%$. GI tract-related AEs reported in mothers treated with pyrimethamine-based therapy were nausea and/or vomiting (21\%) and GI intolerance $(2.3 \%)$ [52, 56].

Across studies, the only dermatologic-related AEs in infants/children were rash, which were reported in four studies $(30.8 \%$ ) (range $0.8-2.1 \%$ ) [48, 51, 55, 58]. Other AEs reported in infants were allergic reaction/hypersensitivity, elevated bilirubin, agitation, multiple blood disorder, and hepatitis. The prevalence of these AEs was $<15 \%$ (range 1.9-13.8\%). Allergic reaction/hypersensitivity $(0.8 \%)$ was observed in mothers who were administered pyrimethamine-based treatment in one study [56].

\subsection{Summary of Adverse Events Across the Three Manifestations of Toxoplasmosis}

The AE profile associated with pyrimethamine-based treatment differed by clinical manifestation of toxoplasmosis. Table 7 summarizes treatment discontinuation/ change and the prevalence of the most common types of AEs. Discontinuation and/or change from pyrimethamine- based treatment was observed in all the included studies for ocular toxoplasmosis (100\%) compared with 57.1 and $61.5 \%$ for TE and congenital toxoplasmosis, respectively. Up to $50 \%$ of patients discontinued or changed therapy because of AEs. Across manifestations, bone marrow suppression and dermatologic AEs were observed in the highest percentage of studies. Bone marrow suppression was lower in studies for ocular toxoplasmosis (36.4\%) than for congenital toxoplasmosis $(76.9 \%)$ and TE $(71.4 \%)$. A smaller percentage of studies in congenital toxoplasmosis reported dermatologic-related AEs $(30.8 \%)$ compared with ocular toxoplasmosis $(63.6 \%)$ and TE (57.1\%). In general, across manifestations, GI-associated AEs were less commonly reported than dermatologic and bone marrow suppression-related AEs. Other AEs reported in single studies, irrespective of manifestation, included abnormal levels of liver enzymes, hepatotoxicity, abnormal alkaline phosphate and pancreatic enzyme levels, elevated bilirubin, allergic reaction/hypersensitivity, agitation, abdominal pain, and malaise.

Across the three manifestations of the disease the most commonly used treatment regimens were pyrimethamine plus sulfadiazine $(n=19)$ and pyrimethamine plus sulfadoxine $(n=6)$. All other regimens were evaluated in only 
one or two studies. Table 8 summarizes treatment discontinuation or change as well as the prevalence of bone marrow suppression, GI, and dermatologic AEs for pyrimethamine plus sulfadiazine and pyrimethamine plus sulfadoxine. A similar percentage of studies reported discontinuation/change in treatment with pyrimethamine plus sulfadiazine $(73.7 \%)$ compared with pyrimethamine plus sulfadoxine (83.3\%). Pyrimethamine plus sulfadiazine compared with pyrimethamine plus sulfadoxine was associated with a lower percentage of studies reporting bone marrow (57.9 vs $83.3 \%$, respectively), GI (31.6 vs $50.0 \%$ ), or dermatologic (42.1 vs $83.3 \%$ ) AEs.

\section{Discussion}

This systematic review of 2975 patients from 31 studies summarizes the current evidence on the prevalence of AEs associated with pyrimethamine-based treatment regimens used to treat the three main manifestations of toxoplasmosis: ocular toxoplasmosis, congenital toxoplasmosis, and TE. The AE profile associated with pyrimethaminebased treatment across the three manifestations has not previously been systematically reviewed.

The types of AEs observed differed across manifestations. AE-related treatment discontinuation or change in therapy occurred in $>55 \%$ of the studies, and up to $50 \%$ of patients discontinued or changed treatment because of AEs. The most commonly observed AEs among the three manifestations were indicative of inhibition of folate metabolism, that is, bone marrow suppression, dermatologic, and GI. A similar percentage of studies in congenital toxoplasmosis and TE reported bone marrow suppression-related AEs, which was higher than the percentage of studies in ocular toxoplasmosis. The number of studies and patients reporting GI disturbances appeared to be more frequent in ocular toxoplasmosis than either TE or congenital toxoplasmosis. Dermatologic AEs appeared to be less frequent in congenital toxoplasmosis than in ocular toxoplasmosis or TE.

The frequency of specific AEs also varied across manifestations. None of the studies in ocular toxoplasmosisof which seven of the 11 were randomized trials-reported neutropenia, while $53.8 \%$ of the studies in congenital toxoplasmosis reported this AE. Eosinophilia was only observed in studies in congenital toxoplasmosis, and pancytopenia was only seen in TE studies. In contrast, thrombocytopenia was observed in studies across all three manifestations. Of the dermatologic AEs, pyrimethaminebased treatment-associated rash was the most commonly reported. The frequency of Steven-Johnson syndrome was uncommon; Steven-Johnson syndrome was reported in three patients $(3 / 2975$ patients; $0.1 \%)$ of which two patients were treated with pyrimethamine plus sulfadoxine for ocular toxoplasmosis and one was treated with pyrimethamine plus sulfadiazine for TE. All three patients were also administered folinic acid. The outcomes of these patients are unknown as they were not reported in the published studies. The most frequently reported GI AEs were diarrhea and nausea/vomiting.

For all manifestations, the most common therapy regimen was pyrimethamine plus sulfadiazine plus folinic acid. Other pyrimethamine-based regimens included sulfadoxine, clindamycin, atovaquone, and TMP-SMX. Compared with pyrimethamine plus sulfadoxine, pyrimethamine plus sulfadiazine was associated with a smaller percentage of studies reporting bone marrow, GI, and dermatologic AEs.

Two studies in TE [44, 45] and five in ocular toxoplasmosis [29, 30, 32-34] assessed the efficacy and safety of non-pyrimethamine-based regimens. These included TMP-SMX and TMP-SMX plus clindamycin, as well as clindamycin and azithromycin monotherapy, clindamycin plus sulfadiazine, and clindamycin plus dexamethasone. For the TMP-SMX-based therapies, reported AEs included neutropenia, diarrhea, minor dermatologic complications, liver impairment, and renal dysfunction in TE $(\leq 22 \%)$ $[44,45]$, and rash in ocular toxoplasmosis (2\%) [33]. In ocular toxoplasmosis, the clindamycin-based therapies were associated with diarrhea, mild hepatotoxicity, skin rash, and thrombocytopenia $(<10 \%)$ [29, 32, 34]. The low number of non-pyrimethamine-based regimens in this systematic review precludes the ability to compare the safety profile of the different therapies with each other and with pyrimethamine-based treatments.

Since in all studies pyrimethamine was used in combination with at least one additional drug, it is not possible to know what specific medication was responsible for a given AE. It is probable that a number of the AEs are not due to pyrimethamine. The reasons for the differences in the prevalence of specific AEs among the included studies are not clear, but likely reflect variation in patient populations (i.e., age, immune status, and disease state), drugs coadministered with pyrimethamine, pyrimethamine pharmacokinetics, treatment protocols, and study design. For example, neutropenia was not observed in patients with ocular toxoplasmosis, possibly reflecting the fact this group of patients were immunocompetent adults. The difference in the prevalence of neutropenia between ocular toxoplasmosis and acute TE studies may also, in part, be due to the higher doses of pyrimethamine used for treating TE. The dosages of pyrimethamine used to treat ocular toxoplasmosis (excluding the loading dose) ranged from 25 to $50 \mathrm{mg} /$ day in all 11 included studies. The dosage of pyrimethamine used to treat acute TE was $75 \mathrm{mg} /$ day in four of the seven studies. The safety profile of pyrimethamine may also be affected by age and body weight, which are 
known to significantly influence pyrimethamine pharmacokinetics possibly through impacting clearance and the volume of distribution $[62,63]$. In addition, pharmacokinetic studies indicate that pyrimethamine serum levels and half-life show wide interpatient variability and differ with age $[62,64,65]$, which may also influence the prevalence of AEs. Additional studies are required to gain further insight into the safety profile of pyrimethamine-based treatment of toxoplasmosis.

Our review has limitations. The number of studies and the total sample size analyzed under each manifestation were low. In addition, specific doses, study designs, and patient populations differed among studies within a given manifestation. The great majority of dosing regimens involved pyrimethamine in combination with another drug and this makes it difficult to know how the individual drugs are related to a given AE. The quality of included studies was fair to poor, the randomized controlled trials did not use blinding of treatments, and cohort studies were included, all of which contribute to a higher probability of information bias. However, the inclusion of both prospective observational and retrospective studies, due to the small number of available randomized studies with pyrimethamine, broadens the range of evidence and may improve the generalizability of the findings.

\section{Conclusion}

This is the first systematic analysis to comprehensively review the safety profile associated with pyrimethaminebased treatments across the three major manifestations of toxoplasmosis. The AE profiles associated with pyrimethamine-based treatments differ by Toxoplasma manifestation. In ocular toxoplasmosis and TE, the most frequent AEs were dermatologic, and in congenital toxoplasmosis, the most frequent AEs were associated with bone marrow suppression. Steven-Johnson syndrome was uncommon and reported in only three patients $(0.1 \%)$. Hematologic AEs occurred in all manifestations and highlight the importance of monitoring the blood of patients administered pyrimethamine-based regimens.

Acknowledgements The authors would like to thank Melissa Giraldo in helping with the literature search.

\section{Compliance with ethical standards}

Funding The study was supported by Turing Pharmaceuticals.

Conflict of interest RBH is currently an employee of Turing Pharmaceuticals. EG is a former employee of Turing Pharmaceuticals. EG is currently affiliated with PTC Therapeutics, Inc., South Plainfield, NJ, USA. JC is a former employee of Turing Pharmaceuticals (former SVP, Medical Affairs).
Open Access This article is distributed under the terms of the Creative Commons Attribution-NonCommercial 4.0 International License (http://creativecommons.org/licenses/by-nc/4.0/), which permits any noncommercial use, distribution, and reproduction in any medium, provided you give appropriate credit to the original author(s) and the source, provide a link to the Creative Commons license, and indicate if changes were made.

\section{References}

1. Dubey JP, Lindsay DS, Speer CA. Structures of Toxoplasma gondii tachyzoites, bradyzoites, and sporozoites and biology and development of tissue cysts. Clin Microbiol Rev. 1998;11(2):267-99.

2. Tenter AM, Heckeroth AR, Weiss LM. Toxoplasma gondii: from animals to humans. Int J Parasitol. 2000;30(12-13):1217-58.

3. Aspöck H. Prevention of congenital toxoplasmosis in Austria: experience of 25 years. Congenital toxoplasmosis. New York: Springer; 2000. p. 277-92.

4. Maenz M, Schluter D, Liesenfeld O, et al. Ocular toxoplasmosis past, present and new aspects of an old disease. Prog Retinal Eye Res. 2014;39:77-106.

5. Halonen SK, Weiss LM. Toxoplasmosis, Chapter 8. In: Hector H, Garcia HBT, Oscar HDB, editors. Handbook of clinical neurology. New York: Elsevier; 2013. p. 125-45.

6. Rabaud C, May T, Amiel C, et al. Extracerebral toxoplasmosis in patients infected with HIV. A French National Survey. Medicine (Baltimore). 1994;73(6):306-14.

7. Jones JL, Holland GN. Annual burden of ocular toxoplasmosis in the US. Am J Trop Med Hyg. 2010;82(3):464-5.

8. Vora NM, Holman RC, Mehal JM, et al. Burden of encephalitisassociated hospitalizations in the United States, 1998-2010. Neurology. 2014;82(5):443-51.

9. Kaye A. Toxoplasmosis: diagnosis, treatment, and prevention in congenitally exposed infants. J Pediatr Health Care. 2011;25(6):355-64.

10. Cuervo G, Simonetti AF, Alegre O, et al. Toxoplasma myocarditis: a rare but serious complication in an HIV-infected late presenter. Aids. 2016;30(14):2253-4.

11. Remington JS, McLeod R, Thulliez P, et al. Toxoplasmosis. In: Remington J, Klein J, editors. Infectious diseases of the fetus and newborn infant. 6th ed. Philadelphia: WB Saunders; 2006. p. 947-1091.

12. Montoya JG, Liesenfeld O. Toxoplasmosis. Lancet. 2004;363(9425):1965-76.

13. Olliaro P. Drug resistance hampers our capacity to roll back malaria. Clin Infect Dis. 2005;41(Suppl 4):S247-57.

14. Khan Assir MZ, Ahmad HI, Akram J, et al. An outbreak of pyrimethamine toxicity in patients with ischaemic heart disease in Pakistan. Basic Clin Pharmacol Toxicol. 2014;115(3):291-6.

15. Beuerman VA, Burnham CJ. Toxoplasmic uveitis; treatment with pyrimethamine and sulfadiazine. Am J Ophthalmol. 1956;42(2):217-27.

16. Lapetina F. Toxoplasmosis and dermatomyositis: a causal or casual relationship? Pediatr Med Chir. 1989;11(2):197-203.

17. Yang Z, Ahn HJ, Park YH, et al. Afatinib reduces STAT6 signaling of host ARPE-19 cells infected with Toxoplasma gondii. Korean J Parasitol. 2016;54(1):31-8.

18. Rajapakse S, Chrishan Shivanthan M, Samaranayake N, et al. Antibiotics for human toxoplasmosis: a systematic review of randomized trials. Pathog Glob Health. 2013;107(4):162-9.

19. Dedicoat M, Livesley N. Management of toxoplasmic encephalitis in HIV-infected adults (with an emphasis on resource-poor settings). Cochrane Database Syst Rev. 2006;3:CD005420. 
20. Hernandez AV, Thota P, Pellegrino D, et al. A systematic review and meta-analysis of the relative efficacy and safety of treatment regimens for HIV-associated cerebral toxoplasmosis: is trimethoprim-sulfamethoxazole a real option? HIV Med. 2017;18(2):115-24.

21. Yan J, Huang B, Liu G, et al. Meta-analysis of prevention and treatment of toxoplasmic encephalitis in HIV-infected patients. Acta Trop. 2013;127(3):236-44.

22. Pradhan E, Bhandari S, Gilbert RE, et al. Antibiotics versus no treatment for toxoplasma retinochoroiditis. Cochrane Database Syst Rev. 2016;5:CD002218.

23. Palella FJ Jr, Delaney KM, Moorman AC, et al. Declining morbidity and mortality among patients with advanced human immunodeficiency virus infection. HIV Outpatient Study Investigators. N Engl J Med. 1998;338(13):853-60.

24. Connolly MP, Goodwin E, Schey C, et al. Toxoplasmic encephalitis relapse rates with pyrimethamine-based therapy: systematic review and meta-analysis. Pathog Glob Health. 2017;2017:1-14.

25. Vidal JE, Oliveira AC. AIDS-related cerebral toxoplasmosis in Sao Paulo State, Brazil: marked improvements in the highly active antiretroviral therapy-era but the challenges continue. Braz J Infect Dis. 2013;17(3):379-80.

26. Mayor AM, Fernandez Santos DM, Dworkin MS, et al. Toxoplasmic encephalitis in an AIDS cohort at Puerto Rico before and after highly active antiretroviral therapy (HAART). Am J Trop Med Hyg. 2011;84(5):838-41.

27. Fayer R, Dubey JP, Lindsay DS. Zoonotic protozoa: from land to sea. Trends Parasitolol. 2004;2004:20.

28. Acers TE, Okla L. Toxoplasmic retinochoroiditis: a double blind therapeutic study. Arch Ophthalmol. 1964;71(1):58-62.

29. Baharivand N, Mahdavifard A, Fouladi RF. Intravitreal clindamycin plus dexamethasone versus classic oral therapy in toxoplasmic retinochoroiditis: a prospective randomized clinical trial. Int Ophthalmol. 2013;33(1):39-46.

30. Balaskas K, Vaudaux J, Boillat-Blanco N, et al. Azithromycin versus sulfadiazine and pyrimethamine for non-vision-threatening toxoplasmic retinochoroiditis: a pilot study. Med Sci Monit. 2012;18(5):Cr296-302.

31. Bosch-Driessen LH, Verbraak FD, Suttorp-Schulten MS, et al. A prospective, randomized trial of pyrimethamine and azithromycin vs pyrimethamine and sulfadiazine for the treatment of ocular toxoplasmosis. Am J Ophthalmol. 2002;2002:34-40.

32. Rothova A, Meenken C, Buitenhuis HJ, et al. Therapy for ocular toxoplasmosis. Am J Ophthalmol. 1993;1993:517-23.

33. Soheilian M, Sadoughi MM, Ghajarnia M, et al. Prospective randomized trial of trimethoprim/sulfamethoxazole versus pyrimethamine and sulfadiazine in the treatment of ocular toxoplasmosis. Ophthalmology. 2005;2005:1876-82.

34. Soheilian M, Ramezani A, Azimzadeh A, et al. Randomized trial of intravitreal clindamycin and dexamethasone versus pyrimethamine, sulfadiazine, and prednisolone in treatment of ocular toxoplasmosis. Ophthalmology. 2011;118(1):134-41.

35. Canamucio CJ, Hallett JW, Leopold IH. Recurrence of treated toxoplasmic uveitis. Am J Ophthalmol. 1963;55:1035-9.

36. Lam S, Tessler HH. Quadruple therapy for ocular toxoplasmosis. Can J Ophthalmol. 1993;28(2):58-61.

37. Borkowski PK, Brydak-Godowska J, Basiak W, et al. The impact of short-term, intensive antifolate treatment (with pyrimethamine and sulfadoxine) and antibiotics followed by long-term, secondary antifolate prophylaxis on the rate of toxoplasmic retinochoroiditis recurrence. PLoS Negl Trop Dis. 2016;10(8):e0004892.

38. Helfenstein M, Zweifel S, Barthelmes D, et al. Ocular toxoplasmosis: therapy-related adverse drug reactions and their management. Klin Monbl Augenheilkd. 2017;234(4):556-60.
39. Bosch-Driessen LH, Verbraak FD, Suttorp-Schulten MS, et al. A prospective, randomized trial of pyrimethamine and azithromycin vs pyrimethamine and sulfadiazine for the treatment of ocular toxoplasmosis. Am J Ophthalmol. 2002;134(1):34-40.

40. Chirgwin K, Hafner R, Leport C, et al. Randomized phase II trial of atovaquone with pyrimethamine or sulfadiazine for treatment of toxoplasmic encephalitis in patients with acquired immunodeficiency syndrome: ACTG 237/ANRS 039 Study. AIDS Clinical Trials Group 237/Agence Nationale de Recherche sur le SIDA, Essai 039. Clin Infect Dis. 2002;2002:1243-50.

41. Podzamczer D, Miro JM, Ferrer E, et al. Thrice-weekly sulfadiazine-pyrimethamine for maintenance therapy of toxoplasmic encephalitis in HIV-infected patients. Spanish Toxoplasmosis Study Group. Eur J Clin Microbiol Infect Dis. 2000;19(2):89-95.

42. Chaddha DS, Kalra SP, Singh AP, et al. Toxoplasmic encephalitis in acquired immunodeficiency syndrome. J Assoc Phys India. 1999;47(7):680-4.

43. Amogne $\mathrm{W}$, Teshager G, Zenebe G. Central nervous system toxoplasmosis in adult Ethiopians. Ethiop Med J. 2006;44(2):113-20.

44. Arens J, Barnes K, Crowley N, et al. Treating AIDS-associated cerebral toxoplasmosis-pyrimethamine plus sulfadiazine compared with cotrimoxazole, and outcome with adjunctive glucocorticoids. S Afr Med J. 2007;97(10):956-8.

45. Goswami RP, Goswami RP, Rahman M, et al. Alternative treatment approach to cerebral toxoplasmosis in HIV/AIDS: experience from a resource-poor setting. Int J STD AIDS. 2015;26(12):864-9.

46. Arendt G, von Giesen HJ, Hefter $\mathrm{H}$, et al. Long-term course and outcome in AIDS patients with cerebral toxoplasmosis. Acta Neurol Scand. 1999;100(3):178-84.

47. McLeod R, Boyer K, Karrison T, et al. Outcome of treatment for congenital toxoplasmosis, 1981-2004: the National Collaborative Chicago-Based, Congenital Toxoplasmosis Study. Clin Infect Dis. 2006;42(10): 1383-94.

48. Guerina NG, Hsu HW, Meissner HC, et al. Neonatal serologic screening and early treatment for congenital Toxoplasma gondii infection. The New England Regional Toxoplasma Working Group. N Engl J Med. 1994;330(26):1858-63.

49. Lipka B, Milewska-Bobula B, Filipek M. Monitoring of plasma concentration of pyrimethamine (PYR) in infants with congenital Toxoplasma gondii infection-own observations. Wiad Parazytol. 2011;57(2):87-92.

50. Schmidt DR, Hogh B, Andersen O, et al. Treatment of infants with congenital toxoplasmosis: tolerability and plasma concentrations of sulfadiazine and pyrimethamine. Eur $\mathrm{J}$ Pediatr. 2006;165(1):19-25.

51. Faucher B, Garcia-Meric P, Franck J, et al. Long-term ocular outcome in congenital toxoplasmosis: a prospective cohort of treated children. J Infect. 2012;64(1):104-9.

52. Hohlfeld P, Daffos F, Thulliez P, et al. Fetal toxoplasmosis: outcome of pregnancy and infant follow-up after in utero treatment. J Pediatr. 1989;115(5 Pt 1):765-9.

53. Mombro M, Perathoner C, Leone A, et al. Congenital toxoplasmosis: 10-year follow up. Eur J Pediatr. 1995;154(8):635-9.

54. Stray-Pedersen B. Infants potentially at risk for congenital toxoplasmosis: a prospective study. Am J Dis Child. 1980;134(7):638-42.

55. Villena I, Aubert D, Leroux B, et al. Pyrimethamine-sulfadoxine treatment of congenital toxoplasmosis: follow-up of 78 cases between 1980 and 1997. Reims Toxoplasmosis Group. Scand J Infect Dis. 1998;30(3):295-300.

56. Hotop A, Hlobil H, Gross U. Efficacy of rapid treatment initiation following primary Toxoplasma gondii infection during pregnancy. Clin Infect Dis. 2012;54(11):1545-52. 
57. Carellos EV, de Andrade JQ, Romanelli RM, et al. High frequency of bone marrow depression during congenital toxoplasmosis therapy in a cohort of children identified by neonatal screening in Minas Gerais, Brazil. Pediatr Infect Dis J. 2017 [Epub ahead of print].

58. Teil J, Dupont D, Charpiat B, et al. Treatment of congenital toxoplasmosis: safety of the sulfadoxine-pyrimethamine combination in children based on a method of causality assessment. Pediatr Infect Dis J. 2016;35(6):634-8.

59. Capobiango JD, Bregano RM, Navarro IT, et al. Congenital toxoplasmosis in a reference center of Parana, Southern Brazil. Braz J Infect Dis. 2014;18(4):364-71.

60. Guerina NG. Congenital infection with Toxoplasma gondii. Pediatr Ann. 1994;23(3):138-42, 147-51.

61. Teil J, Dupont D, Charpiat B, et al. Treatment of congenital toxoplasmosis: safety of the sulfadoxine-pyrimethamine combination in children based on a method of causality assessment. Pediatr Infect Dis J. 2016;35(6):634-8.

62. Barnes KI, Little F, Smith PJ, et al. Sulfadoxine-pyrimethamine pharmacokinetics in malaria: pediatric dosing implications. Clin Pharmacol Ther. 2006;80(6):582-96.
63. Trenque T, Simon N, Villena I, et al. Population pharmacokinetics of pyrimethamine and sulfadoxine in children with congenital toxoplasmosis. Br J Clin Pharmacol. 2004;57(6):735-41.

64. Corvaisier S, Charpiat B, Mounier C, et al. Population pharmacokinetics of pyrimethamine and sulfadoxine in children treated for congenital toxoplasmosis. Antimicrob Agents Chemother. 2004;48(10):3794-800.

65. Klinker H, Langmann P, Richter E. Plasma pyrimethamine concentrations during long-term treatment for cerebral toxoplasmosis in patients with AIDS. Antimicrob Agents Chemother. 1996;40(7):1623-7.

66. Couvreur J, Nottin N, Desmonts G. Treatment of congenital toxoplasmosis. Clinical and biological results (author's transl). Ann Pediatr. 1980;27(10):647-52.

67. Schmidt DR, Hogh B, Andersen O, et al. The national neonatal screening programme for congenital toxoplasmosis in Denmark: results from the initial four years, 1999-2002. Arch Dis Child. 2006;2006:661-5.

68. Stray-Pedersen B. A prospective study of acquired toxoplasmosis among 8,043 pregnant women in the Oslo area. Am J Obstet Gynecol. 1980;136(3):399-406. 